\title{
PTU-056 QUALITY OF LIFE IN PATIENTS UNDERGOING SMALL INTESTINAL TRANSPLANTATION
}

doi:10.1136/gut.2011.239301.184

C Pither, ${ }^{*}$ A Wiles, ${ }^{1}$ A Butler, ${ }^{2}$ H Tinknell, ${ }^{1}$ S Duncan, ${ }^{2}$ J Woodward, ${ }^{1}$ N Jamieson, ${ }^{2}$ B Chukualim, ${ }^{1}$ M Manaligod, ${ }^{1}$ S Gabe, ${ }^{3}$ S Middleton ${ }^{1}$ Gastroenterology, Cambridge University Hospitals NHS Foundation Trust, Cambridge, UK; ${ }^{2}$ Transplantation Surgery, Cambridge University Hospitals NHS Foundation Trust, Cambridge, UK; ${ }^{3}$ Gastroenterology, St Marks Hospital, London, UK

Introduction Intestinal transplantation is currently considered for patients with irreversible intestinal failure who develop life threatening complications of home parenteral nutrition (HPN) or who have extensive intra-abdominal disease requiring evisceration

Thus far quality of life (QOL) has not been used as an indication for transplantation but as post-operative survival improves this may be considered appropriate in the future if improvements in QOL can be obtained compared with HPN. Previous studies suggest improvement in some aspects in carefully selected patients. ${ }^{1-3}$

Our latest 2 year survival rates are 100\% (non-oncological) and $98 \%$ (oncological). We looked at changes in the QOL of this cohort of recent transplantations

Aims To assess the quality of life of patients requiring small intestinal transplantation using the SF 36 tool.

Methods 18 patients completed the questionnaire as part of the transplant assessment and subsequently at 6 monthly intervals.

Data was scored using validated criteria for each of eight functions: physical function, role functioning-physical, role functioning- emotional, energy/fatigue, emotional wellbeing, social functioning, pain and general health.

Results A total number of 36 datasets were analysed, 19 preoperatively (6 male, 13 female) and 17 post-operatively from a total of 8 patients ( 4 male, 4 female). Paired scores pre and postoperatively from 6 patients ( 3 male, 3 female) were also analysed.

The grouped data showed a significant improvement in physical function $p=0.05^{*}$ social function $p=0.01^{*}$ and general health $\mathrm{p}=0.05^{*}$. Other function scores were very similar.

General health was the only modality showing significant improvement in the paired data $\mathrm{p}=0.03^{* *}$. When the data was analysed for each patient, 2 out of 6 patients had significantly better overall scores after the transplant, $\mathrm{p}=0.02^{* *}, \mathrm{p}=0.007^{* *}$. *Mann-Whitney U test ** Wilcoxon signed rank

Conclusion QOL on HPN is impaired ${ }^{4} 5$ and declines over time. Our short term survival is approaching that of HPN Some aspects of QOL can be improved with transplantation but others do not seem to improve. If $\mathrm{QOL}$ is to be a factor in the consideration of patients for intestinal or multivisceral transplantation it will be important to look at each individual quality of life aspect as some seem to be more likely to improve than others. Longer term studies are required

Competing interests None.

\section{REFERENCES}

1. Cameron E. et al Transplant Proceedings 2002;34:965-66

2. DiMartini A et al. JPEN 1998;22(6):357-62.

3. O'Keefe S et al. Am J Gastroenterology 2007;102(5):1093-1100.

4. Jeppenson et al Gut 1999;44:844.

5. ichards DM et al. Gut 1997;40:218. 